\title{
Premio Pusterla 2021
}

\section{Tobias Eichinger}

Dr. phil., Institut für Biomedizinische Ethik und Medizingeschichte, Universität Zürich

Bereits zum vierten Mal konnten sich im vergangenen Herbstsemester an der Universität Zürich die über 400 Erstsemesterstudierenden der Human- und Zahnmedizin an dem Essaywettbewerb «Premio Pusterla Medizinethik» beteiligen. Nachdem sich die Studienanfängerinnen und -anfänger an drei Nachmittagen in Kleingruppen im Rahmen eines kompakten Einführungskurses mit ethischen Aspekten des medizinischen Tätigkeitsfeldes auseinandergesetzt hatten, galt es, die obligatorische Lehrveranstaltung mit dem Verfassen eines kurzen Aufsatzes abzuschliessen, der der Darstellung und Diskussion eines ethisch herausfordernden Falles im klinischen oder praktischen Kontext gewidmet war. So hatten die Studierenden gleich zu Beginn ihres Studiums Gelegenheit, schwierige moralische und zum Teil persönlich fordernde Fragen, die sich in ihrem künftigen Beruf typischerweise stellen können und die sich nicht mit Faktenwissen alleine beantworten lassen, in einer schriftlichen Reflexion zu erörtern und, soweit jeweils möglich, einen entsprechenden Lösungsvorschlag zu erarbeiten. Dabei sollten die im Kurs vermittelten Inhalte noch einmal anhand eines Praxisbeispiels angewendet und in ihrer Relevanz für den ärztlichen Alltag verdeutlicht werden.

Viele Studierende wählten für diese Kurztexte Fallgeschichten, zu denen sie einen persönlichen Bezug hatten, erzählten von Patientinnen und Patienten aus ihrem näheren Umfeld, die in Krisensituationen auf medizinische Hilfe angewiesen und dabei mit manchmal unklaren oder schwierigen Situationen konfrontiert waren. In manchen Aufsätzen wurde spürbar, dass das Verfassen für die Autorin oder den Autor mehr war als die Erfüllung einer Pflichtaufgabe und dass die schriftliche Darstellung und Reflexion ein besonderes Engagement bei den Erstsemestrigen wachgerufen hatte. Bei einigen blitzte nicht nur eine ganz persönliche Note auf, in der die Studienwahl und der Berufswunsch, später als Ärztin oder Arzt Menschen zu helfen, zum Ausdruck kamen, auch die Freude am Formulieren und In-Sprache-Bringen des Allzumenschlichen fand sich in dem einen oder anderen Pflichtaufsatz. So war es dann für einen verhältnismässig grossen Anteil des Semesters ein stimmiges Folgeangebot, im Anschluss an die Vorlesungszeit den eigenen Text noch einmal zu überarbeiten und für den Essay-Wettbewerb einzureichen.

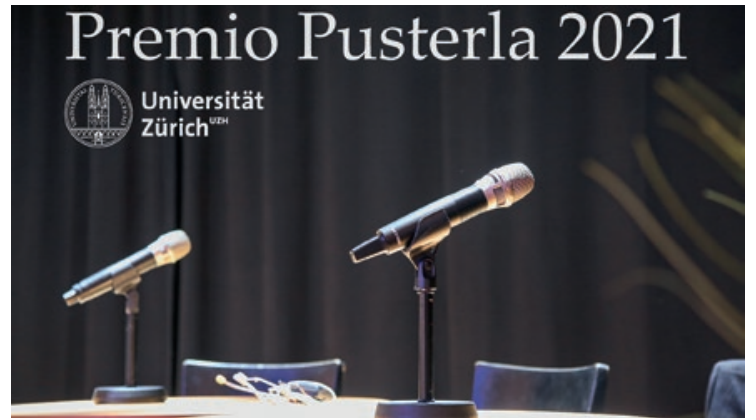

Schon zum zweiten Mal musste das Wettbewerbsfinale online stattfinden.

Die Preisträgerinnen des Premio Pusterla Junior 2021 sind: Lotte Habermeyer mit "Danke, dass Du anders bist», 1. Platz (auf Seite 1108-1109 dieser Ausgabe);

Seraphina Limacher mit "Das Recht auf den Tod», 2. Platz (online unter saez.ch $\rightarrow$ Ausgabe 34/2021);

Ornella Scandella mit «Entscheidung für ein Leben», 3. Platz (online unter saez.ch $\rightarrow$ Ausgabe 34/2021).

Der aktuelle Jahrgang war ein besonders wertvoller, da mit über 50 Texten so viele Ersteinreichungen eingingen wie nie zuvor; es waren darüber hinaus vor allem auch bemerkenswert viele Essays von beeindruckender Qualität dabei. So konnten die elf besten Texte für die zweite Runde ausgewählt werden. Nun hatten die Studierenden Gelegenheit, in einem persönlichen Schreib-Coaching weiter an ihrem Text zu feilen, Unebenheiten zu glätten und das Ergebnis erneut einzugeben. Von einer Fachjury, die medizinisch-fachliche mit literarisch-schriftstellerischer Expertise sowie einer Patientenperspektive vereinte (Melitta Breznik, Brida von Castelberg, Frank Clasemann, Anna Elsner, Christoph A. Meier), wurden daraufhin in einem anonymisierten Verfahren die drei Essays bestimmt, denen es nach Einschätzung der Jurorinnen und Juroren am besten gelungen war, relevante medizinethische Themen in einer ansprechenden sprachlichen Form zu präsentieren.

Es konnten sich drei Studentinnen durchsetzen, die am öffentlichen Finale des Wettbewerbs teilnahmen, das pandemiebedingt erneut online stattfand. Die Autorinnen trugen ihre Texte per Videoübertragung vor, woraufhin die Jurymitglieder und das zahlreich zugeschaltete Publikum ihre Voten abgeben konnten. 